

\title{
Non-autonomous Ginzburg-Landau solitons using the He-Li mapping method
}

Pérez Maldonado, Maximino; C. Rosu, Haret; Flores Garduño, Elizabeth

Non-autonomous Ginzburg-Landau solitons using the He-Li mapping method CIENCIA ergo-sum, Número especial "Retos de la física no lineal" 2020|e104

Universidad Autónoma del Estado de México, México

Esta obra está bajo una Licencia Creative Commons Atribución-NoComercial-SinDerivar 4.0 Internacional .

Pérez Maldonado, M., C. Rosu, H. y Flores Garduño, E. (2020). Non-autonomous Ginzburg-Landau solitons using the He-Li mapping method. CIENCIA ergo-sum, Número especial "Retos de la física no lineal". https:// doi.org/10.30878/ces.v27n4a3 


\title{
Non-autonomous Ginzburg-Landau solitons using the He-Li mapping method
}

\section{Solitones Ginzburg-Landau no autónomos mediante el método de mapeo de He-Li}

\author{
Maximino Pérez Maldonado \\ Universidad Politécnica de San Luis Potosí, México \\ maximino.perez@upslp.edu.mx \\ (D) https://orcid.org/0000-0002-6785-2203 \\ Haret C. Rosu \\ Instituto Potosino de Investigación Cientifica y Tecnológica, México \\ hcr@ipicyt.edu.mx \\ (1) https://orcid.org/0000-0001-5909-1945 \\ Elizabeth Flores Garduño \\ Instituto Potosino de Investigación Cientifica y Tecnológica, México \\ elizabeth.flores@ipicyt.edu.mx \\ (1) https://orcid.org/0000-0002-5715-0347
}

Recepción: 24 de junio de 2019

Aprobación: 17 de febrero de 2020

\begin{abstract}
We find and discuss the non-autonomous soliton solutions in the case of variable nonlinearity and dispersion implied by the Ginzburg-Landau equation with variable coefficients. In this work we obtain non-autonomous Ginzburg-Landau solitons from the standard autonomous Ginzburg-Landau soliton solutions using a simplified version of the He-Li mapping. We find soliton pulses of both arbitrary and fixed amplitudes in terms of a function constrained by a single condition involving the nonlinearity and the dispersion of the medium. This is important because it can be used as a tool for the parametric manipulation of these non-autonomous solitons.
\end{abstract}

KEYWORDS: nonlinear, Ginzburg-Landau Equation, Non-Autonomous Solitons.

\section{RESUMEN}

Se hallan y discuten soluciones de tipo solitones no autónomos en el caso de no linealidad y dispersión implícitas en la ecuación de Ginzburg-Landau con coeficientes variables. El principal objetivo del artículo es obtener de manera sistemática las soluciones de dicha ecuación mediante una versión simplificada del mapeo propuesto por $\mathrm{He}-\mathrm{Li}$ a partir de las soluciones solitónicas autónomas de la ecuación de Ginzburg-Landau estándar de coeficientes constantes. Bajo este mapeo, se encuentran pulsos solitonicos de amplitudes tanto fijas como arbitrarias que dependen de una función que es restringida por una única condición que involucra la no linealidad y la dispersión del medio. Esté resultado es importante porque puede usarse como una herramienta para la manipulación paramétrica de solitones no autónomos.

Palabras ClaVe: no lineal, ecuación de Ginzburg-Landau, solitones no autónomos.

\section{INTRODUCTION}

As it is well known, dispersion and dissipation are extremely important for soliton pulse propagation in nonlinear media. These two processes are the main cause for the distortion and losses of the signal (Agrawal, 2001; Hasegawa, \& Matsumoto, 2003; Ablowitz, Prinari, \& Trubatch, 2004), and have been studied since the end of 
1960's, although it was not until 1980's that people began to use amplifiers to compensate those losses (Hasegawa, \& Matsumoto, 2003). In the amplification process, the silica doping of fibers is commonly used (Ablowitz, Prinari, \& Trubatch, 2004).

Theoretically, this kind of propagation is mainly described by the nonlinear Schrödinger equation (NLS), but other equations can be used, such as the Sine-Gordon, Korteweg-de Vries, and Ginzburg-Landau equations that can also describe this kind of soliton propagation (Agrawal, 2001; Hasegawa, \& Matsumoto, 2003; Ablowitz, Prinari, \& Trubatch, 2004).

Nowadays, there are significant advances in the description of pulses in nonlinear media and the way they can be manipulated. The NLS equation with variable coefficients, and its non-autonomous nonlinear dynamical systems form (Malomed, 2006; He \& Li, 2011; Pérez-Maldonado, \& Rosu, 2015) are very important in this context of variable dispersion and nonlinearity, which bring losses and gains during the propagation. The manipulation of these pulses for optimal propagation is usually called "soliton management", or also for its specific use in optical devices as "dispersion management" (Malomed, 2006; Porsezian et al., 2007; Centurion et al., 2006).

In the case of non-autonomous models, the soliton management is defined by four parameters (Zhao, Luo, \& He, 2010): a) amplitude (or width), b) frequency (or velocity), c) phase and $d$ ) time position. It is possible to control the soliton dynamics with a careful selection of their functional form of these parameters.

In this paper, we work out an extension of the method of non-autonomous NLS management (Pérez-Maldonado, \& Rosu, 2015) to the Ginzburg-Landau equation (GL), employing both fixed amplitudes and arbitrary ones.

Compared to NLS, the GL equation has smaller damped terms and considerably bigger nonlinear terms (Malomed, 1991), and thus it governs the amplitude evolution of the dissipative waves in finite spatial neighborhoods over instabilities close to the critical points of the singularities (Akhmediev \& Ankiewicz, 2005).

\section{The NON AUTONOMOUS MODEL}

Soliton interaction between NLS non-autonomous solitons has been studied in a systematic way by Serkin and coworkers (Serkin, \& Hasegawa, 2000; Serkin, \& Belyaeva, 2001; Serkin \& Hasegawa, 2002; Serkin, Hasegawa, \& Belyaeva, 2004) who have been the pioneers in the discussion of the non-autonomous solitons (Serkin, Hasegawa, \& Belyaeva, 2007). They proved that the non-autonomous solitons can propagate within nonlinear media when their amplitude and velocity are controlled (Serkin, Hasegawa, \& Belyaeva, 2007).

The NLS equation governing the propagation of an optical soliton through an optical material with engineered dispersion and nonlinearity is

$$
i \frac{\partial \psi(z, t)}{\partial z}+f(z) \quad \frac{\partial^{2} \psi(z, t)}{\partial t^{2}}+g(z)|\psi(z, t)|^{2} \psi(z, t)+i \gamma(z) \psi=0 .
$$

where $f(z)$ and $g(z)$ are the dispersion and nonlinearity management, respectively, and $\gamma(z)=\gamma_{\text {loss }}+\gamma_{R}$, with $\gamma_{\text {loss }}>0$, the constant loss parameter of the fiber, and $\gamma_{R}<0$ is the Raman gain parameter. If the functions $f(z)$, $g(z)$, and $\gamma(z)$ are complex functions, then equation (1) is known as the complex nonlinear Ginzburg-Landau equation (CGL) (Fang \& Xiao, 2006).

We now apply the He-Li mapping (He \& Li, 2011) of transforming the NLS equation with variable coefficients (NLS-CV) to a standard NLS equation of constant coefficients to the case of pulse propagation in doped fiber amplifiers for which the one-dimensional cubic CGL equation (Agrawal, 1991)

$$
i \frac{\partial \psi}{\partial z}+\left(p_{r}(z)+i p_{i}(z)\right) \frac{\partial^{2} \psi}{\partial t^{2}}+\left(q_{r}(z)+i q_{i}(z)\right)|\psi|^{2} \psi=i\left(\gamma_{r}(z)+i \gamma_{i}(z)\right) \psi
$$


is considered as a good model. Here $\psi(z, t)$ is the field envelope function, $t$ is the retarded time and $z$ the propagation distance, $p_{r}$ measures the wave dispersion, $p_{i}$ the spectral filtering $\left(p_{i}<0\right)$ of the amplifier, which is important because it suppresses the Gordon-Haus jitter of the soliton central frequency; ${ }^{[1]} q_{r}$ and $q_{i}>0$ represent the nonlinear coefficient and the nonlinear gain/absorption processes, respectively. We noticed that the nonlinear gain helps to suppress the growth of radiative background (linear mode) which always affects the propagation of nonlinear stationary pulses in real fiber links; $\gamma_{r}$ and $\gamma_{i}$ are the linear gain and the frequency shift, respectively (Fewo et al., 2005). The parameters $p_{r}, p_{i}, q_{r}, q_{i}, \gamma_{r}$, and $\gamma_{i}$ are real functions depending on the propagation coordinate. In many cases, this type of model is used for dispersion-managed solitons (DMS) (Biswas, 2002; Hasegawa, 2000) in transmission lines employing a periodic map, such that each period is built up by two types of fibers of generally different lengths and opposite group velocity dispersion (Turitsyn $e t$ al., 2003; Turitsyn, 1998; Turitsyn et al., 1999). On the other hand, a too strong dispersion management could lead to system performances even worse than the conventional soliton systems (Hirooka, Nakada, \& Hasegawa, 2000).

Previously, some exact solutions were obtained by other methods. For example, the stability of chirped bright and dark soliton-like solutions of the cubic CGL equation with variable coefficients has been investigated in (Fang, \& Xiao, 2006), but here we will use a modified He-Li mapping (He \& Li, 2011; Pérez-Maldonado, \& Rosu, 2015).

\section{1. Autonomous mapPing OF THE NON-AUTONOMOUS SOLITONS IN AMPLIFIED DisSiPATIVE FIBERS}

Firstly, we should look for the integrability of the equation (2) by finding a direct relationship with the standard GL equation (Akhmediev \& Afanasjev, 1995; Soto-Crespo et al., 1997) (without quintic term), given by

$$
i \frac{\partial \psi}{\partial z}+\left(\frac{D}{2}-i \beta\right) \frac{\partial^{2} \psi}{\partial t^{2}}+(s-i \varepsilon)|\psi|^{2} \psi=i \delta \psi,
$$

where $\psi$ is the envelope of the optical field, and $z$ and $t$ are the propagation distance and retarded time, respectively. The parameters in (3) are all real parameters, namely, $\delta$ stands for linear gain or loss, $\beta$ for spectral filtering, and $\varepsilon$ for nonlinear gain. The parameters $D$ and $s$ may only take the values \pm 1 , i.e., when $D=1$ the dispersion is anomalous and when $D=-1$ the dispersion is normal, and $s=1$ or $s=-1$ stands for positive or negative Kerr effect (Facao \& Carvalho, 2015), respectively. The proposed ansatz is similar to the Talanov ansatz (Talanov, 1970),

$$
\psi(t, z)=u(T, Z) e^{-i \phi(t, z)}
$$

where the phase function describes the instability (Hasegawa, \& Matsumoto, 2003). With $T=T(z, t)$ and $Z$ $=Z(z)$, the central task is to determine the specific expressions for $p_{r}, p_{i}, q_{r}, q_{i}, \gamma_{r}, \gamma_{i}, \phi, T$, and $Z$ by requesting $u(T, Z)$ to satisfy the standard autonomous GL equation (3). It is important to mention that for rare-earth doped optical fibers, which are normally used by people for pulse amplification with retarded time $T=t-\beta_{1}$ eff $z$ (Agrawal, 1991), where $\beta_{1}$ eff is related to the effective group velocity, we have a $T$-dependent $Z$. Therefore, by derivating the ansatz (4) and taking into account that $\partial \psi / \partial t=\psi_{t}$ and $\partial \psi / \partial z=\psi_{z}$, we obtain

$$
i u_{z}+\left(\frac{D}{2}-i \beta\right) u_{T T^{+}}+(s-i \varepsilon)|u|^{2} u+\left(k_{1}+i k_{2}\right) u_{T^{+}}+\left(k_{3}+i k_{4}\right) u=i \delta u,
$$
from where one can see the following relationships between the parameters $p_{r}(z)=\frac{D}{2} \frac{Z_{z}}{T_{t}^{2}}, p_{i}(z)=-\beta \frac{Z_{z}}{T_{t}^{2}}$,
$q_{r}(z)=s Z_{z}, q_{i}(z)=-\varepsilon Z_{z}$ y $\gamma_{r}(z)=\delta Z_{z}$, with

$$
k_{1}=\frac{D}{2} \frac{T_{t t}}{T_{t}^{2}}-2 \beta \frac{\phi_{t}}{T_{t}}
$$




$$
\begin{gathered}
k_{2}=\frac{T_{z}}{z_{z}}-\beta \frac{T_{t t}}{T_{t}^{2}}-D \frac{\phi_{t}}{T_{t}} \\
k_{3}=\frac{\phi_{z}}{Z_{z}}-\beta \frac{\phi_{t t}}{T_{t}^{2}}-\frac{D}{2} \frac{\phi_{t}^{2}}{T_{t}^{2}}+\frac{\gamma_{i}(z)}{z_{z}}, \\
\delta=\beta \frac{\phi_{t}^{2}}{T_{t}^{2}}-\frac{D}{2} \frac{\phi_{t t}}{T_{t}^{2}} .
\end{gathered}
$$

When $k_{1}=k_{2}=k_{3}=0$, we have the standard cubic CGL equation (3). For the complete description, we need to solve the latter equations to find the specific functional form of each of the mentioned terms.

Taking $T=t-\beta_{1}^{e f f} z$

$$
\begin{gathered}
T_{t}=\frac{\partial T}{\partial \xi} \frac{d \xi}{d t}=T_{\xi} \\
T_{z}=\frac{\partial T}{\partial \xi} \frac{d \xi}{d z}=-\beta_{1}^{e f f} T_{\xi}
\end{gathered}
$$

and substituting into (9)

$$
\begin{gathered}
k_{1}=\frac{D}{2} \frac{T_{\xi \xi}}{T_{\xi}^{2}}-2 \beta \frac{\phi_{t}}{T_{\xi}}, \\
k_{2}=\frac{-\beta_{1}^{e f f} T_{\xi}}{z_{z}}-\beta \frac{T_{\xi \xi}}{T_{\xi}^{2}}-D \frac{\phi_{t}}{T_{\xi}}, \\
k_{3}=\frac{\phi z}{z z}-\beta \frac{\phi_{t t}}{T_{\xi}^{2}}-\frac{D}{2} \frac{\phi_{t}^{2}}{T_{\xi}^{2}}, \\
\delta=\frac{\gamma_{r}(z)}{z_{z}}-\beta \frac{\phi_{t}^{2}}{T_{\xi}^{2}}+\frac{D}{2} \frac{\phi_{t t}}{T_{\xi}^{2}},
\end{gathered}
$$

we can solve this system of equations, obtaining the following results

$$
\begin{gathered}
T(z, t)=F_{1}(z)+\frac{\sqrt{s . c}}{k} \sqrt{g(z)} \sqrt{2 k(t-\omega z)+f_{1}}, \\
Z(z)=(s . c) \int g(z) d z+f_{2}, \\
\phi(z, t)=-\frac{D}{8 \beta} \ln \left(2 k(t-\omega z)+f_{1}\right),
\end{gathered}
$$

where $f_{1}$ and $f_{2}$ are integration constants, $s . c$ and $D . c$ are mapping complex quantities of the form s. $c=\frac{s+i \epsilon}{s^{2}+\epsilon^{2}}$ and D. $c=\frac{\frac{D}{2}+i \beta}{\frac{D^{2}}{2}+\beta^{2}}$. These complex numbers are related to the nonlinear and dispersion 
coefficients of the autonomous GL equation, respectively; also, $\omega=k / 8 \beta\left(16 \beta^{2}+D^{2}\right)$ and $\kappa=\beta_{1}^{e f f}\left(\frac{4 \beta}{D^{2}+\beta^{2}}\right)$, and so we can obtain the transformation function $F_{1}(z)$

$$
F_{1}(z)=\left(\frac{1}{k}+\frac{4 \beta_{1}^{e f f}}{\omega}\right) \sqrt{\frac{S . c}{D \cdot c}} \sqrt{g(z) p(z)} .
$$

Therefore, we can express the function for the loss/gain with dependence on nonlinearity and dispersion in the form

$$
\gamma(z)=s . c g(z)-\frac{3 D^{2} k^{2}}{16 \beta p(z)}
$$

so that we are able to obtain the integrability of the system.

\section{EXACT SOLUTION FOR CGL EQUATION OF STANDARD COEFFICIENTS}

There exist some exact solutions of the equation (3) (Akhmediev \& Afanasjev, 1995; Soto-Crespo et al., 1997; Facao \& Carvalho, 2015; Conte \& Musette, 1995) and for our mapping method we can use any of these solutions. In this work, we make use only of the solution proposed by Soto-Crespo et al. (1997) for bright solitons with anomalous dispersion $D=s=1$. This is because in the anomalous dispersion regime it is possible to study the main properties of the soliton-like solutions of equation (3) by applying the well-developed soliton perturbation theory of the NLS equation. There are two different solutions proposed by Soto-Crespo et al. (1997), one is for solitons with fixed amplitude and the other is for solitons with arbitrary amplitude. In both cases, the soliton solution has the form

$$
u(T, Z)=a(T) \exp [i d \ln [a(T)]-\omega Z]
$$

where $a(T)$ is a real function, and $d$ and $\omega$ are real constants with values

$$
\begin{gathered}
d_{ \pm}=\frac{3(1+2 \varepsilon \beta D) \pm \sqrt{9(1+2 \varepsilon \beta D)^{2}+8(\varepsilon-2 \beta D)^{2}}}{2(\varepsilon-2 \beta D)} \\
\omega=\frac{\delta\left(1-d^{2}-4 \beta d D\right)}{2\left(d-\beta D+\beta d^{2} D\right)} .
\end{gathered}
$$

We also consider the special stability conditions as proposed by Soto-Crespo et al. (1997).

\section{1. The soliton with fixed amplitude}

The solution for $a(T)$ is in this case

$$
a(T)=C B \operatorname{sech}(B T),
$$

where

$$
B=\sqrt{\frac{\delta}{\beta d^{2}+D d-\beta}}
$$




$$
C=\sqrt{\frac{3 d\left(1+4 \beta^{2}\right.}{2(2 \beta-\varepsilon D)}}
$$

and $d$ is given by equation (20) after choosing the plus (minus) sign in front of the square root if $D$ is negative (positive). The second value of $d$ does not lead to a physical solution (Akhmediev \& Afanasjev, 1995; Akhmediev, Afanasjev, \& Soto-Crespo, 1996), as the expression under the square root for $C$ becomes negative.

Soto-Crespo et al. (1997) also provided the following condition of existence of the solution (22),

$$
\varepsilon_{S}=\beta \frac{\sqrt[3]{1+4 \beta^{2}-D}}{4+18 \beta^{2}}
$$

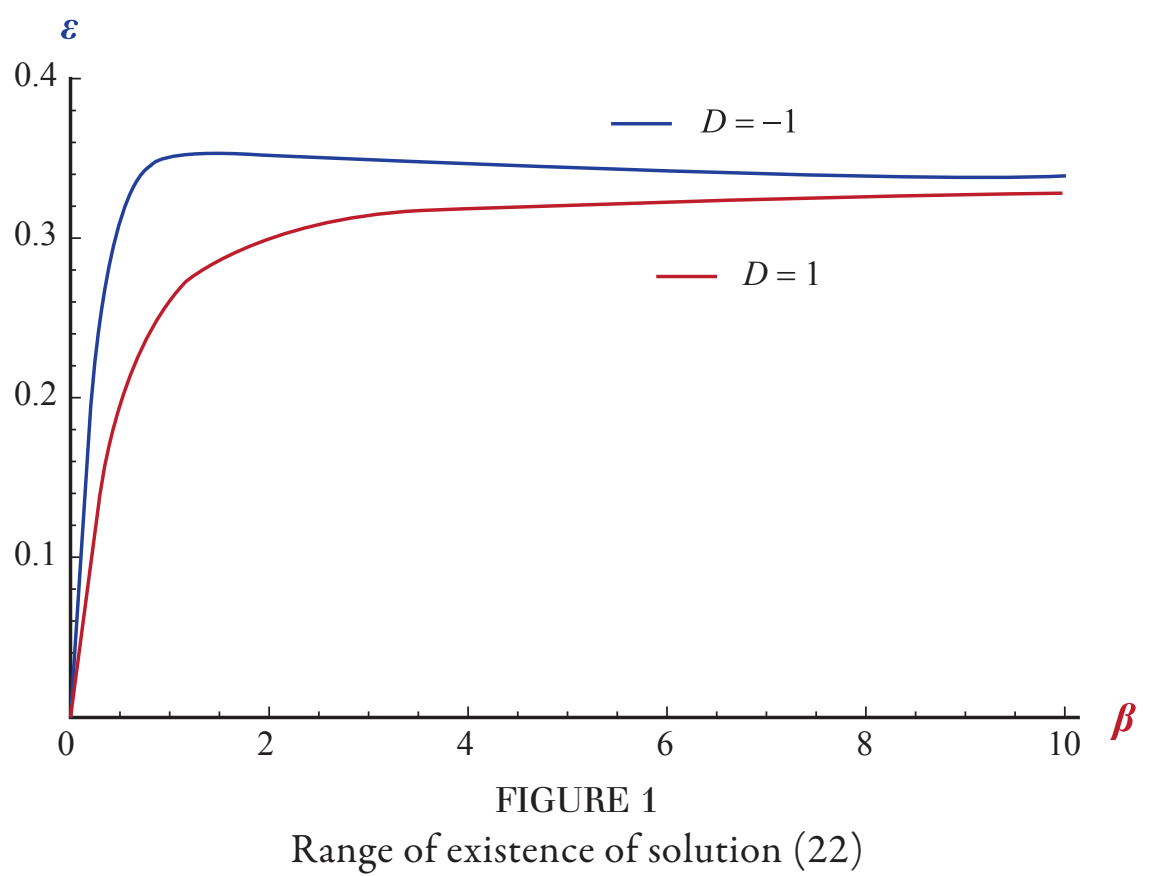

Nota: The curve $S(25)$ in the plane $(\varepsilon, \beta)$ where the solutions with fixed amplitudes (22) become singular, and where the classes of special solutions with arbitrary amplitude (26) exist. This plot applies for cubic and quintic cases. The corresponding one for the case of anomalous dispersion is also shown by the blue curve for comparison. Above the curve $S, \delta$ must be positive for solution (22) to exist, and negative below it. The red curve depicts $S$ for $D=+1$, similar to obtained by Soto-Crespo et al. (1997).

As this solution exists almost everywhere in the $(\varepsilon, \beta)$ plane, we call it the general solution (Figure 1). The curve $S$ itself is the line where this solution becomes singular, i.e., its amplitude BC tends to infinity, while the width $1 / B$ vanishes.

\section{2. The solution with arbitrary amplitude}

Another special solution proposed by Soto-Crespo et al. (1997) is obtained if we also impose the condition $\delta=0$. Then, the solution, valid only on the line (25), is

$$
a(T)=G F \operatorname{sech}(G T),
$$

where $G$ is an arbitrary positive parameter, and $d, \omega$ and $F$ are given by

$$
d=\frac{\sqrt{1+\beta^{2}}-D}{2 \beta}
$$




$$
\begin{gathered}
\omega=-\frac{\left(1+4 \beta^{2}\right)\left(\sqrt{1+4 \beta^{2}}-D\right)}{4 \beta^{2}} G^{2}=-d \frac{\sqrt{1+\beta^{2}}-D}{2 \beta} G^{2} \\
F=\left(\frac{d \sqrt{1+\beta^{2}}-D}{2 \varepsilon}\right)^{1 / 2}=\left[\frac{\left(2+9 \beta^{2}\right) \sqrt{1+\beta^{2}}\left(\sqrt{1+\beta^{2}}-D\right)}{2 \beta^{2}\left(3 \sqrt{1+\beta^{2}}-D\right)}\right]^{1 / 2} .
\end{gathered}
$$

The solution (26) represents the arbitrary amplitude soliton. The reason for the existence of the arbitrary amplitude solutions is that, when $\delta=0$, the cubic CGL equation becomes invariant relative to the scaling transformation $u \rightarrow G u, T \rightarrow G T, Z \rightarrow G^{2} Z$. Hence, if we know a particular solution of this equation, the whole family can be generated using this transformation. Notice that all parameters of solution (26) except $G$ and the coefficient $\varepsilon$ are expressed in terms of $\beta$.

\section{Solution of THE CGL With VARIABLE COEFFicients}

We will now use a specific case for the CGL equation with variable coefficients employed in the work of Fang and Xiao (2006), to describe a fiber with inhomogeneities. They found solutions for chirped, bright and dark solitons. Here, we work with their dispersion parameter and solve their CGL equation by the mapping method.

First, we introduce their dispersion and nonlinearity parameters

$$
\begin{gathered}
p(z)=-\frac{1}{2} p_{0}\left[1+\alpha_{1} \sin (\sigma z)\right] \operatorname{ex} p\left(-\mu_{1} z\right), \\
g(z)=g_{0}\left[1+\alpha_{2} \sin (\sigma z)\right] \exp \left(-\mu_{2} z\right),
\end{gathered}
$$

where $p_{r ; 0}$ and $q_{r} ; 0$ are ideal fiber parameter; $\alpha_{1}$ is a smal parameter that characterizes the amplitude of the fluctuations; $\mu_{1}$ is a small constant; is related to the variation period of the fiber parameters. In our case, we only need the dispersion parameter and the frequency shift parameter $\gamma_{i}(z)$ is set equal zero, so and we can solve the problem analytically.

Now, taking into account all parameters, we obtain the complete solution, with the $T$ equation (14), $Z$ equation (15) and $\phi$ equation (16) for both cases, where from (17)

$$
F_{1}(z)=\left(\frac{1}{k}+\frac{4 v}{\omega}\right) \sqrt{\frac{S \cdot c}{D \cdot c}} \sqrt{-\frac{1}{2} p_{0} g_{0}\left[1+\alpha_{2} \sin (\sigma z)\right] \exp \left(-\mu_{2} z\right)\left[1+\alpha_{1} \sin (\sigma z)\right] \exp \left(-\mu_{1} z\right)}
$$

It is possible to define more solutions, one is for bright solitons with $\beta \delta>0$ and another for dark solitons when $\beta \delta<0$.

\section{1. The soliton solution for fixed amplitude}

In accordance with the proposed solution by Soto-Crespo et al. (1997) our solution is

$$
\psi(t, z)=C B \operatorname{sech}(B T) \exp [i(d \ln [a(T)]-\omega Z-\phi(t, z))]
$$

which is displayed graphically in Figure 2. 

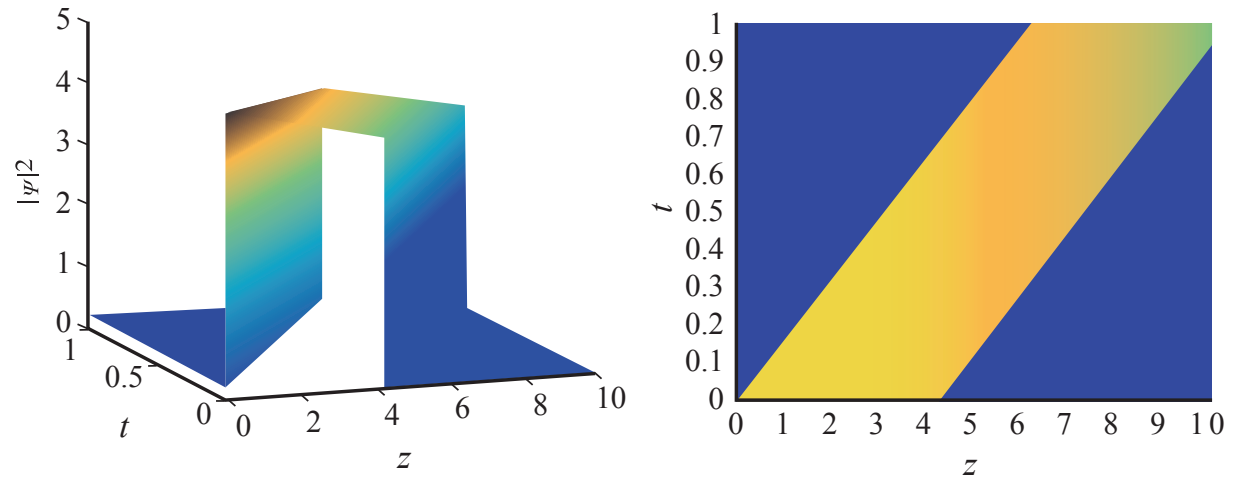

FIGURE 2

Non-autonomus soliton with fixed amplitude

Nota: a) Soliton solution with fixed amplitude for bright solitons obtained from (33). b) Contour plot.

Figure 2 shows the graphs for the soliton solution of the nonlinear CGL equation with variable coefficients with anomalous dispersion, i. e. the coefficient $D=1$. We note that our solutions present similarities with the solutions reported by Soto-Crespo et al. (1997). The values of the parameters are the following: $\beta=0.25, \delta=-0.1, \alpha_{1}$ $=0.05, \alpha_{2}=0.1, \sigma=0.05, \mu_{1}=\mu_{2}=0.01, p_{0}=-0.5, g_{0}=0.3$, and $s=1$, likewise those of Soto-Crespo et al. (1997) and Fang, \& Xiao (2006).

\section{2. The soliton solution with arbitrary amplitude}

On the other hand, we obtain the following soliton solution with arbitrary amplitude,

$$
\psi(t, z)=G F \operatorname{sech}(G T) \exp [i(d \ln [a(T)]-\omega Z-\phi(t, z))]
$$

with its graphic representation in the figure (3), where we have used the following values of the parameters: $G$ $=1, \beta=0.1, \alpha_{1}=0.05, \alpha_{2}=0.1, \sigma=0.05, \mu_{1}=\mu_{2}=0.01, p_{0}=-0.5, g_{0}=0.3$, and $s=1$.


FIGURE 3

Non-autonomus soliton with arbitrary amplitude

Note: a) Bright soliton of arbitrary amplitude obtained from (34).b) Contour plot.

Examination of the obtained solution in equation (19) shows that it is scalable in the amplitude and the variable $T(z, t)$, whereas the scaling in the $Z(z)$ variable produces only a change of the phase of the soliton solution, so essentially the solution (19) can be considered as scalable. The importance of the scaling properties lies in the 
possibility to obtain a whole family of solutions once a particular solution is known, just by varying the scaling parameter. In our case, each member of the family of solutions will have a different phase. Physically, changes in the scaling parameter can be achieved by varying the power of the source (laser) of the pulses.

Furthermore, we can notice that under this transformation, the propagation function only depends on the nonlinearity, as expected, because the nonlinearity is a characteristic of the medium where the pulse is propagating. Indeed, the function $T(z, t)$ has a direct dependence on the nonlinearity of the medium, but also depends on the traveling variable and the dispersion of the medium as one can see from equation (14). This is what assures the management of solitons through the compensation of the dispersion and nonlinearity functions according to equation (18).

\section{Prospective}

The mathematical treatment uses the simplified mapping of the He-Li method as a way to find solutions of nonlinear equations of variable coefficients, commonly used in nonlinear optics for modeling the propagation of solitons in nonlinear media.

The method can be implemented for other nonlinear equations different from the ones treated here, taking into account that it is only necessary to find the transformation equation that takes us to the mapping itself, where we can use all the solutions of autonomous nonlinear equations already known.

\section{Conclusion}

Using a modified He-Li mapping approach we have been able to obtain the appropriate conditions that assure the system integrability for the management of non-autonomous solitons in nonlinear media, such as, fiber optics or waveguides, for the nonlinear Ginzburg-Landau equation with variable coefficients. In addition, it is possible to use all known solutions of the autonomous Ginzburg-Landau equation, because, in our development we find a transformation function that maps the GL equation of variable coefficients to the autonomous GL equation, as well as we can use the stability conditions of the GL equation presented in the literature.

\section{REFERENCES}

Ablowitz, M. J., Prinari, B., \& Trubatch, A. (2004). Discrete and continuous nonlinear Schrödinger systems. Cambrige University Press. https://doi.org/10.1007/b11728.

Agrawal, G. P. (1991). Optical pulse propagation in doped fiber amplifiers, Phys. Rev. A., 44(11), 7493 7501. https://doi.org/10.1103/PhysRevA.44.7493.

Agrawal, G. P. (2001). Nonlinear fibers optics. Elsevier Science.

Akhmediev, N., \& Afanasjev, V. (1995). Novel arbitrary-amplitude soliton solutions of the cubic-quintic complex Ginzburg-Landau equation. Phys. Rev. Lett., 75(12), 2320-2324. https://doi.org/10.1103/ PhysRevLett.75.2320.

Akhmediev, N., Afanasjev, V., \& Soto-Crespo, J. M. (1996). Singularities and special soliton solutions of the cubic-quintic complex Ginzburg-Landau equation. Phys. Rev. E., 53(1), 1190-1201. https:// doi.org/10.1103/PhysRevE.53.1190.

Akhmediev, N. \& Ankiewicz, A. (2005). Adrian (Eds.), Dissipative Solitons, Lecture Notes in Physics, 661. Springer-Verlag Berlin Heidelberg. 
Biswas, A. (2002). Dispersion-managed solitons in optical fibers, J. Optics A., 4(1), 84-97. https://doi. org/10.1088/1464-4258/4/1/315.

Centurion, M., Porter, M. A., Kevrekidis, P. G. \& Psaltis, D. (2006). Nonlinearity management in optics: Experiment, theory, and simulation, Phys. Rev. Lett., 97, 033903-1-033903-4. https://doi. org/10.1103/PhysRevLett.97.033903.

Conte, R., \& Musette, M. (1995). Exact solutions to the complex Ginzburg-Landau equation of nonlinear optics. Pure Appl. Opt., 4, 315-320. https://doi.org/10.1088/0963-9659/4/4/005.

Facao, M., \& Carvalho, M. I. (2015). Existence and stability of solutions of the cubic complex GinzburgLandau equation with delayed Raman scattering. Phys. Rev. E., 92, 022922-1-022922-5. https:// doi.org/10.1103/PhysRevE.55.4783.

Fang, F., \& Xiao, Y. (2006). Stability of chirped bright and dark soliton-like solution of cubic complex Ginzburg-Landau equation with variable coefficients. Optics Communications, 268, 305-310. https://doi.org/10.1016/j.optcom.2006.07.014.

Fewo, S., Atangana, J., Kenfack-Jiotsa, A., \& Kofane, T. (2005). Dispersion-managed solitons in the cubic complex Ginzburg-Landau equation as perturbations of nonlinear Schrödinger equation. Opt. Comm., 252, 138-149. https://doi.org/10.1016/j.optcom.2005.03.031.

Hasegawa, A. (2000). Massive WDM and TDM Soliton Transmission Systems. Kluwer Academic Publishers.

Hasegawa, A., \& Matsumoto, M. (2003). Optical Solitons in Fibers. Springer.

He, J., \& Li, Y. (2001). Designable integrability of the variable coefficient nonlinear Schrödinger equations. Stud. Appl. Math., 126, 1-15. https://doi.org/10.1111/j.1467-9590.2010.00495.x.

Hirooka, T., Nakada, T. \& Hasegawa, A. (2000). Feasibility of densely dispersion managed soliton transmission at $160 \mathrm{~Gb} / \mathrm{s}$. IEEE Photon. Technol. Lett. 12(6), 633-635. https://doi. org/10.1109/68.849067.

Malomed, B. A. (1991). Bound solitons in the Nonlinear Schrödinger/Ginzburg-Landau equation. In J. D. Fournier, \& P. L. Sulem (Eds.), Large Scale Structures in Nonlinear Physics. Lecture Notes in Physics, 392. Springer, Heidelberg. Springer-Verlag.

Malomed, B. (2006). Soliton Management in Periodic Systems. Springer. https://doi.org/ 10.1007/0-387-29334-5.

Pérez-Maldonado, M. \& Rosu, H. (2015). Solitones no autónomos en fibras ópticas. Rev. Mex. Fis., 61, 351-355. https://rmf.smf.mx/ojs/rmf/article/view/4161

Porsezian, K., Hasegawa, A., Serkin, V., Belyaeva, T., \& Ganapathy, R. (2007). Dispersion and nonlinear management for femtosecond optical solitons. Phys. Lett. A, 361, 504-508. https://doi.org/10.1016/j. physleta.2006.10.022.

Serkin, V. N., \& Hasegawa, A. (2000). Novel soliton solutions of the nonlinear Schrödinger equation model. Phys. Rev. Lett., 85, 4502-4505. https://doi.org/10.1103/PhysRevLett.85.4502.

Serkin, V. N., \& Belyaeva, T. (2001). High-energy optical Schrödinger solitons. JETP Letters, 74, 573576. https://doi.org/10.1134/1.1455063

Serkin, V. N. \& Hasegawa, A. (2002). Exactly integrable nonlinear Schrödinger equation models with varying dispersion, nonlinearity and gain: Application for soliton dispersion. IEEE J. Sel. Top. in Quantum Electron. 8, 418-431. https://doi.org/10.1109/JSTQE.2002.1016344.

Serkin, V. N., Hasegawa, A., \& Belyaeva, T. (2004). Comment on exact self-similar solutions of the generalized nonlinear Schrödinger equation with distributed coefficients. Phys. Rev. Lett., 92, 199401-1. https://doi.org/10.1103/PhysRevLett.92.199401. 
Serkin, V. N., Hasegawa, A. \& Belyaeva, T. (2007). Nonautonomous solitons in external potentials. Phys. Rev. Lett., 98, 074102-1-074102-4. https://doi.org/10.1103/PhysRevLett.98.074102.

Soto-Crespo, J. M., Akhmediev, N. N., Afanasjev, V. V., \& Wabnitz, S. (1997). Pulse solutions of the cubic-quintic complex Ginzburg-Landau equation in the case of normal dispersion. Phys. Rev. E., 55(4), 4783-4796. https://doi.org/10.1103/PhysRevE.55.4783

Talanov, V. (1970). Focusing of light in cubic media. JETP Lett., 11, 199-201. Retrieved from http:// www.jetpletters.ac.ru/ps/1719/article26119.pdf

Turitsyn, S. (1998). Theory of guiding-center breathing soliton propagation in optical communication systems with strong dispersion management, in new trends in optical soliton transmission systems. A. Hasegawa (Ed.). Kluwer Academic Publishers, Dordrecht, The Netherlands, 225-243. https://doi. org/10.1364/OL.22.001544

Turitsyn, S. K., Doran, N. J., Nijhof, J. H. B., Mezentsev, V. K., Shaefer, T., \& Forysiak, W. (1999). Dispersion-Managed Solitons, in Theoretical Challenges and Industrial Perspectives. V. E. Zakharov, \& S. Wabnitz, S. (Eds. Kluwer.). Academic Publishers, Dordrecht, The Netherlands. Retrieved from https://www.lpsm.paris/pageperso/garnier/publi/reviewsolitonoptics.pdf

Turitsyn, S. K., Shapiro, E. G., Medvedev, S. B., Fedoruk, M. P., \& Mezentsev, V. K. (2003). Physics and mathematics of dispersion-managed optical solitons. Comptes Rendus Physique, Acad'emie des sciences/'Editions scientifiques et m'edicales, 4, 145-161.

Zhao, D., Luo, H.-G., \& He, X.-G. (2010). On the nonautonomous nonlinear Schrödinger equations and soliton management. AIP Conf. Proc., 1212, 213-218. https://doi.org/10.1063/1.3367048.

\section{Notes}

[1] The Gordon-Haus jitter effect refers to the fact that the fluctuations of the central frequencies of the optical pulses are temporally coupled through the group velocity dispersion: a change of the central frequency is presented as a change of the group velocity dispersion, which affects the time coordinate of the pulse.

\section{BY-NC-ND}

